\title{
Relación entre estrés académico y consumo de alcohol y tabaco en estudiantes de educación superior
}

\author{
Márquez-Granados Karla Lizbeth*, Tavarez-Reyes Diana Laura*, Flores-Tapia Miriam del Carmen*,
} Carreón-Rangel María Paola*, Macías-Galaviz María Teresa**, Rodríguez-Ramírez José Manuel**

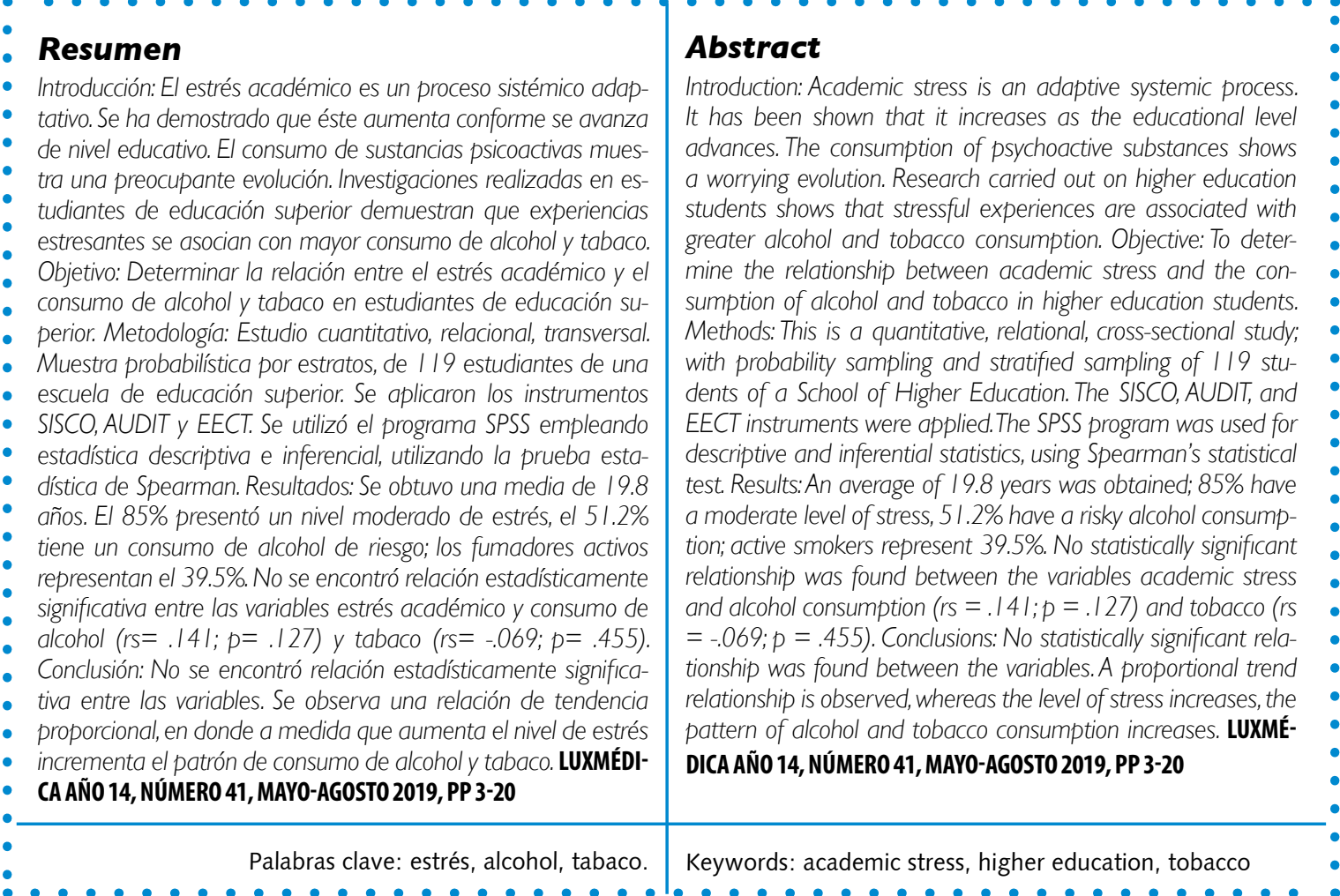

Estudiante de la Licenciatura en Enfermería del Centro de Ciencias de la Salud, Departamento de Enfermería de la Universidad Autónoma de Aguascalientes

** Profesor investigador del Departamento de Enfermería del Centro de Ciencias de la Salud, de la Universidad Autónoma de Aguascalientes

Fecha de recibido: 18 de septiembre 2018

Fecha de aceptación: 10 de enero 2019

Correspondencia: MSPS Ma. Teresa Macías Galaviz. Departamento de Enfermería del Centro de Ciencias de la Salud. Ciudad Universitaria. Universidad Autónoma de Aguascalientes. Avenida Universidad 940. Código postal 20131. Teléfono: 4492127194. Aguascalientes, Aguascalientes, México. Correo electrónico: tmg2699@hotmail.com 


\section{Introducción}

El estrés académico se ha definido como un proceso sistémico, de carácter adaptativo esencialmente psicológico, que se presenta de manera descriptiva en tres momentos; el primero, cuando el alumno se ve sometido, en contextos escolares, a una serie de demandas que, bajo la valoración del propio alumno son consideradas estresores; el segundo, cuando esos estresores provocan un desequilibrio sistémico (situación estresante) que se manifiesta en una serie de síntomas (indicadores del desequilibrio); y el tercero, cuando ese desequilibrio sistémico obliga al alumno a realizar acciones de afrontamiento para restaurar el equilibrio sistémico. ${ }^{1} \mathrm{Se}$ ha estudiado cómo el estilo de vida de los estudiantes se ve modificado según se acerca el periodo de trabajos complejo y exámenes, convirtiéndose así sus hábitos en insalubres, lo que posteriormente puede llevar a la aparición de trastornos de salud. ${ }^{2}$ Algunos estudios han demostrado que ciertas experiencias altamente estresantes se asocian con un mayor consumo de drogas como el alcohol y tabaco en los adolescentes que las han vivido. ${ }^{3}$

El fenómeno del consumo, abuso y dependencia de sustancias psicoactivas legales o ilegales muestra una preocupante evolución. Sus principales indicadores revelan un incremento de las prevalencias de consumo, así como una disminución en las edades de inicio. ${ }^{4}$ El consumo de alcohol se define como aquel consumo crónico y continuado o consumo periódico de alcohol que se caracteriza por un deterioro del control sobre la bebida, episodios frecuentes de intoxicación y obsesión por el alcohol y su consumo a pesar de sus consecuencias adversas. ${ }^{5}$

En México, uno de los principales problemas de salud se encuentra vinculado al uso nocivo del alcohol, mismo que se asocia a importantes problemas de salud en general. Este fenómeno ha mostrado un incremento principalmente en el grupo de la población más joven del país, lo que implica importantes consecuencias. ${ }^{4}$

En México, en la Encuesta Nacional de Consumo de Drogas, Alcohol y Tabaco 2016-2017 (ENCODAT) se informó que el 71.0\% de las personas de 12 a 65 años ha consumido alcohol alguna vez, mientras que el $33.6 \%$ tuvo un consumo excesivo en el último año y un $19.8 \%$ en el último mes. ${ }^{6}$ El tabaco es un problema mundial de salud pública. Sus consecuencias son numerosas, desde enfermedades como cáncer, enfermedad pulmonar obstructiva crónica, enfermedades cardiovasculares y cerebrovasculares. ${ }^{7}$ Se entiende consumo de tabaco como la acción y efecto de consumir tabaco, adicción que produce una dependencia física y psicológica a la nicotina del tabaco. ${ }^{8}$ De acuerdo con la ENCODAT 2016-2017, en México se reporta que 14.9 millones de mexicanos son fumadores actuales de los cuales 6.4 millones fuman diariamente y 9.4 millones fuman de forma ocasional. ${ }^{9}$

En una investigación similar realizada por Villegas Pantoja y cols, se encontró que la media de edad de inicio en el consumo de 


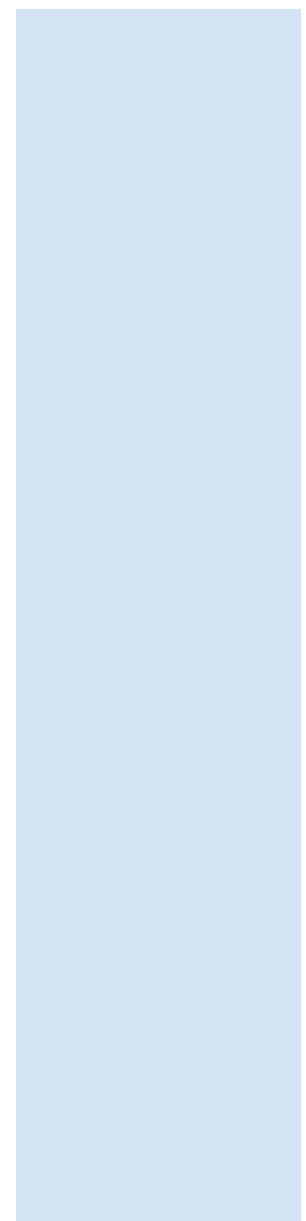

sustancias se ubica por debajo de los 15 años. El evento estresante relacionado con los estudios se presentó en un $67.5 \%$, se encontró que el promedio de bebidas alcohólicas consumidas en un día típico fue de 3.7 copas lo cual indica un consumo de riesgo y éste se presentó en un $73.8 \%$; las prevalencias de tabaco de alguna vez en la vida fueron de $36.3 \%$, y se presentó una correlación positiva entre los eventos estresantes y el consumo de alcohol y tabaco. ${ }^{3}$

Catillo Ávila y cols, con la finalidad de obtener la relación que existe entre el estrés generado por la carga académica en los estudiantes y el consumo de alcohol de éstos, descubrieron que en relación con el estrés, el $90.8 \%$ tuvo momentos de preocupación o nerviosismo, el $42.0 \%$ reportó un nivel moderado de estrés, el $48.2 \%$ manifestó que la sobrecarga de tareas escolares era la principal fuente de estrés seguida de la evaluación del profesor con un $41.7 \%$. En relación con el consumo de alcohol, se encontró que un $65.8 \%$ han consumido alguna bebida alcohólica y el $46.8 \%$ consume alcohol de una o menos veces al mes; también se descubrió que el $50.7 \%$ presenta riesgo de consumo de alcohol, un $79.0 \%$ de los estudiantes no presenta estos síntomas y acerca del consumo perjudicial de alcohol $83.9 \%$ no presentan estas consecuencias. Al concluir la investigación no se encontró una relación significativa entre el estrés académico y el consumo de alcohol, pero se menciona que entre más aumenta la carga de tareas o actividades escolares aumenta el consumo de alcohol. ${ }^{10} \mathrm{El}$ objetivo del presente estudio fue determinar la relación que existe entre el estrés académico y el consumo del alcohol y tabaco en estudiantes de educación superior.

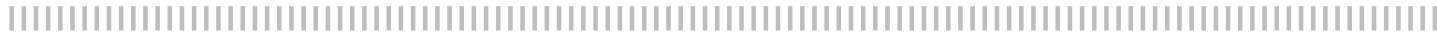

\section{Material y Métodos}

Estudio con enfoque cuantitativo, alcance relacional, no experimental, transversal. Realizado en estudiantes de una Escuela de Educación Superior de Aguascalientes, en el periodo de septiembre a diciembre de 2017. El universo estuvo conformado por 173 estudiantes. Se utilizó un muestreo probabilístico por estratos con remplazo, con una confiabilidad del $95 \%$ y un margen de error del $5 \%$. El tamaño de la muestra fue de 119 estudiantes. La distribución de los estudiantes quedó de la siguiente manera: primer semestre con 39 estudiantes, tercer semestre con 31, quinto semestre con 26 y séptimo semestre con 24 .

Se consideró como criterios de inclusión que fueran estudiantes de educación superior independientemente del semestre en el que se encontraran y que aceptaran participar en el estudio.

Las variables sociodemográficas fueron la edad, semestre y estado civil, la variable independiente fue el estrés académico y las variables dependientes fueron el consumo de alcohol y el consumo de tabaco.

Para la recolección de datos de la variable estrés académico se utilizó el Inventario SISCO del Estrés Académico (Anexo I), creado por Arturo Barraza Macías, con un alfa de Cronbach de 0.87, tiene 34 ítems, el tiempo aproximado para su llenado es de 15 minutos. El instrumento cuenta con 5 apartados en los que se evalúan diferentes aspectos: filtro (1 ítem, respuesta dicotómica si-no); nivel de intensidad del estrés académico (1 ítem, escala de Likert del 0-4 donde cero es nunca y cuatro, siempre); 
dimensión de estresores (9 ítems, escala de Likert de 0-4 donde cero es nunca y cuatro, siempre); dimensión de síntomas (15 ítems, escala de Likert de 0-4 donde cero es nunca y cuatro siempre) y la dimensión estrategias de afrontamiento (8 ítems, escala de Likert de 0-4 donde cero es nunca y cuatro, siempre).

Interpretación general: se toma en cuenta los ítems de las preguntas tres, cuatro y cinco; se obtiene la media por cada ítem y posteriormente se obtiene la media general. Se transforma la media en porcentaje, multiplicando la media obtenida por 25. Una vez obtenido el porcentaje se interpreta con el siguiente baremo: $0-33 \%$ nivel leve, $34-66 \%$ nivel moderado, 67 $-100 \%$ nivel profundo. ${ }^{11}$

Para la recolección de datos de la variable consumo de alcohol se utilizó el instrumento AUDIT (Alcohol Use Disordes Identification Test) (Anexo II) desarrollado por la OMS, con un alfa de Cronbach de 0.86 , tiene 10 ítems, el tiempo aproximado de llenado fue de 5-10 minutos, la interpretación del instrumento es a mayor ponderación mayor dependencia. En cada ítem, el valor de las respuestas oscila entre 0 y 4 (en los ítems 9 y 10 los valores posibles son tan sólo 0,2 y 4). Puntuaciones de $0=$ no consumidor, 1-5=consumo de riesgo de alcohol, $6-20=$ consumo perjudicial, mayor de $20=$ dependencia $^{5,12}$

Para la recolección de datos del consumo de tabaco se utilizó el instrumento denominado "Escala Exploratoria de Consumo de Tabaco" (EECT) (Anexo III). Tiempo de llenado de 5 a 10 minutos. Este instru- mento evalúa la prevalencia y patrones de consumo de tabaco. La EECT constituye un estrato del utilizado en la ENA 2008 y también incluye la Escala de Fagerstrom. Con un alfa de Cronbach de 0.97. ${ }^{13,14}$

La recolección de datos se realizó a través de la aplicación de los instrumentos antes mencionados, con horarios establecidos por equipo de investigadores y los directivos de la institución. Los investigadores se dividieron en dos equipos y se aplicó simultáneamente a los grupos, se dio instrucciones al grupo acerca del llenado, y al finalizar se realizó una revisión detallada de los instrumentos. Se asistió a la escuela en tres ocasiones para el Ilenado. El tiempo promedio de llenado de los instrumentos es de 30 minutos.

Los datos obtenidos se capturaron y analizaron estadísticamente en el programa SPSS versión 22.0, aplicando estadística descriptiva como medidas de tendencia central y medidas de dispersión, y estadística inferencial. Se utilizó la prueba estadística coeficiente de correlación rho de Spearman para comprobar la hipótesis.

La investigación fue aprobada por la academia de investigación del Departamento de Enfermería del Centro de Ciencias de la Salud de la Universidad Autónoma de Aguascalientes, el código de registro es AIE-30-17; se basó en el Reglamento de la Ley General de Salud en materia de investigación para la salud (Artículo 13, 14, $16,17,18,20$ y 21) y en la Declaración de Helsinki de la Asociación Médica Mundial (principios básicos). ${ }^{15,16}$

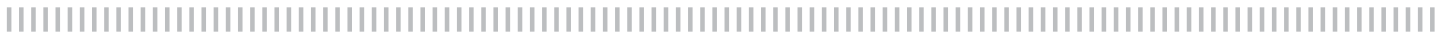

\section{Resultados}

\section{Características sociodemográficas}

Se evaluó una muestra de 119 estudiantes de una escuela de educación superior, de los cuales el $100 \%$ correspondió al sexo femenino, el rango de edad fue de 11 años, una moda de 18 años, con un mínimo de
17 años y un máximo de 28 años, y una media de 19.8 años (DE \pm 1.94). La distribución de la frecuencia del estado civil de las estudiantes fue $91.59 \%$ solteras, $6.72 \%$ casadas y $1.68 \%$ se encontraba en unión libre. En cuanto a la distribución de los alumnos por semestre el $32.77 \%$ pertenecía a primer semestre, el $26.05 \%$ a ter- 
cer semestre, el $21.84 \%$ a quinto semestre y el $19.32 \%$ a séptimo semestre.

\section{Estrés académico}

El nivel de estrés académico moderado fue el encontrado con mayor frecuencia, con un 85\% ( $n=71$ estudiantes) (Gráfica 1). El total de la muestra de estudiantes informó haber tenido momentos de preocupación o nerviosismo durante el transcurso del semestre; de ellas, el 37.80\% reportó tener casi siempre y $31.90 \%$, algunas veces.

\section{Grafica I}

\section{Nivel de estrés académico}

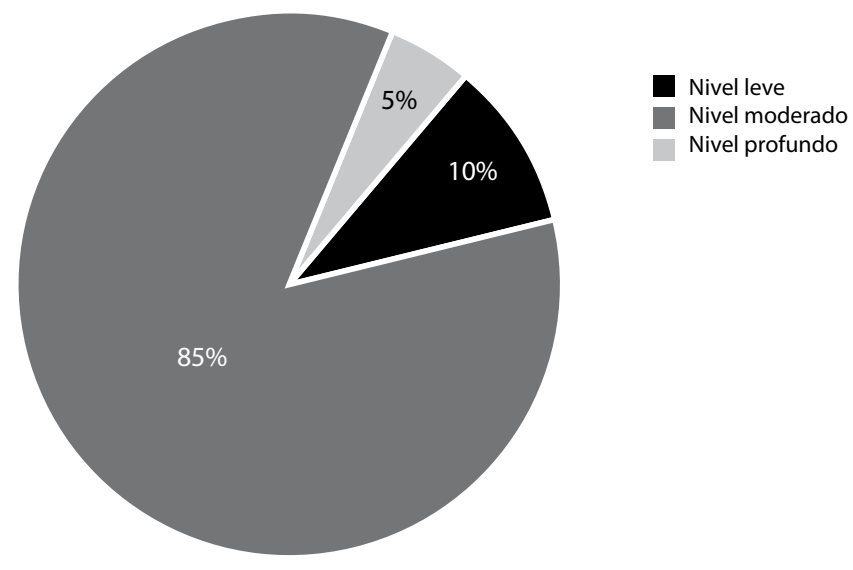

FUENTE: Relación entre estrés académico y consumo de alcohol y tabaco en estudiantes de educación superior $(n=119)$, septiembre-diciembre 2017.

En cuanto a la dimensión de estresores, $75.84 \%$ de las alumnas manifestó que el factor que mayor estrés genera es la evaluación del profesor (exámenes, ensayos, trabajos de investigación), seguido de la sobrecarga de tareas con $74.58 \%$, mientras que el factor que menos le causó estrés fue la competencia entre compañeros con $35.08 \%$.

La dimensión síntomas tiene tres subdimensiones:

- Reacciones físicas: la más frecuente fue la somnolencia o mayor necesidad de dormir con $54.60 \%$, seguido del dolor de cabeza y migraña $53.60 \%$, la menos frecuente fue los problemas digestivos, dolor abdominal y diarrea con $37.20 \%$.

- Reacciones psicológicas: la más frecuente fue los problemas de concentración con $51.10 \%$, seguido de la ansiedad, angustia o desesperación con $48.50 \%$, mientras que la reacción menos frecuente fue la agresividad e irritabilidad con $39.90 \%$.

- Reacciones comportamentales: la más frecuente fue el aumento o reducción del consumo de alimentos con $45.20 \%$ y la menos frecuente fue el aislamiento con $26.50 \%$.

En cuanto a la dimensión de estrategias de afrontamiento, la estrategia que con mayor frecuencia utilizan es concentrarse en resolver la situación que les preocupa 
con un $71.80 \%$ y la estrategia menos frecuente fue la religiosidad con un $36.10 \%$.

\section{Consumo de alcohol}

Se encontró que el $79.80 \%$ de las estudiantes consume bebidas alcohólicas y el
$20.20 \%$ manifestó nunca haber consumido alcohol. De las 95 estudiantes que se consideraron consumidoras, por lo menos 61 de ellas presentan un patrón de consumo de riesgo (Gráfica 2).

\section{Grafica 2}

\section{Patrón de consumo de alcohol}

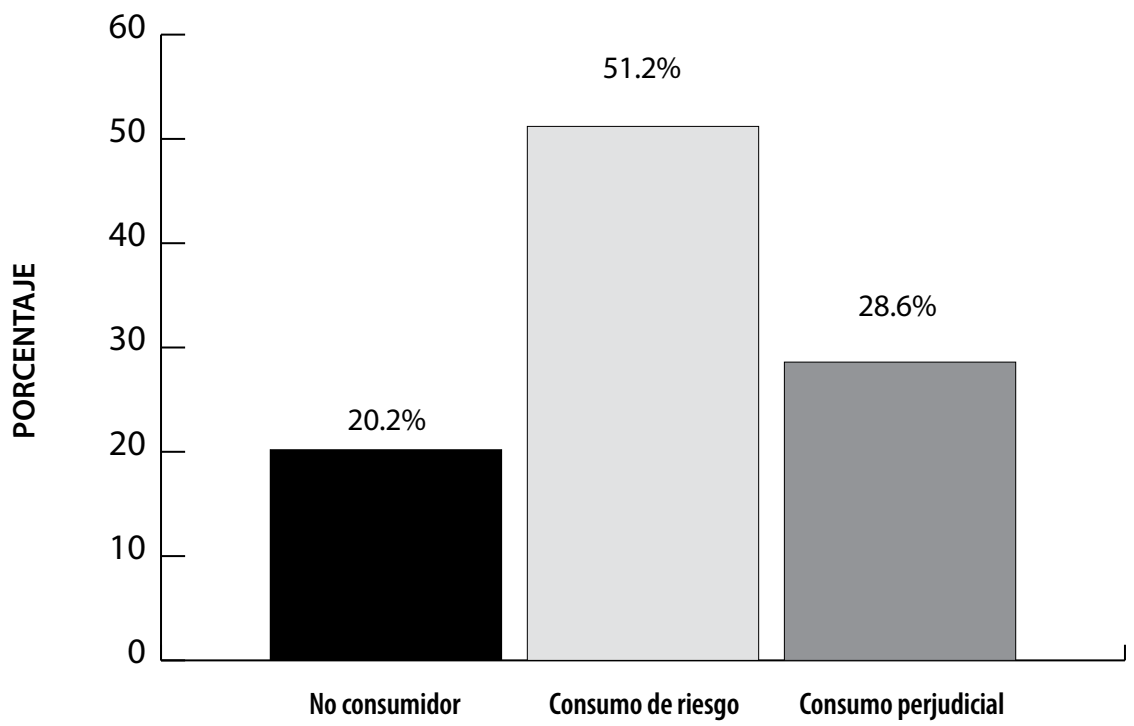

FUENTE: Relación entre estrés académico y consumo de alcohol y tabaco en estudiantes de educación superior ( $n=119$ ), septiembre-diciembre 2017.

De las estudiantes que consumen alcohol, $38.70 \%$ consume una vez al mes, $33.60 \%$ consume de 2 a 4 veces al mes; de acuerdo con el número de bebidas en un día de consumo normal $45.30 \%$ consume de 3 a 4 bebidas, $52.60 \%$ consume 6 bebidas o más en un solo día al mes, $28.40 \%$ tiene la necesidad de beber en ayunas para recuperarse después de haber bebido de- masiado el día anterior, $85.3 \%$ refiere no tener incapacidad para realizar actividades cotidianas porque habían bebido.

\section{Consumo de tabaco}

De la población estudiada, 47 alumnas representaban a los fumadores activos, 20 a los exfumadores y 52 a los no fumadores (Tabla I). 


\section{Tabla I}

Distribución porcentual de la Encuesta exploratoria de consumo de tabaco

\begin{tabular}{|c|c|c|}
\hline \multicolumn{3}{|l|}{ ENCUESTA EXPLORATORIA DE CONSUMO DE TABACO } \\
\hline PREGUNTAS & RESPUESTA & PROCENTAJE \\
\hline \multirow[t]{3}{*}{ Distribución de la población estudiada según consumo de tabaco } & Fumadores activos & 39.5 \\
\hline & Exfumadores & 16.8 \\
\hline & No fumadores & 43.7 \\
\hline \multirow[t]{3}{*}{ Prevalencia de consumo de tabaco } & Alguna vez en la vida & 56.3 \\
\hline & En el último año & 39.5 \\
\hline & En el último mes & 20.9 \\
\hline \multirow[t]{4}{*}{ Edad de inicio de consumo } & 14 años & 5.9 \\
\hline & 15 años & 9.2 \\
\hline & 16 años & 14.3 \\
\hline & 17 años & 25.2 \\
\hline \multirow[t]{2}{*}{ Ha intentado dejar de fumar } & Sí & 36.1 \\
\hline & No & 19.3 \\
\hline \multirow[t]{2}{*}{ Frecuencia de consumo de tabaco } & Semanalmente & 5.9 \\
\hline & Ocasionalmente & 33.6 \\
\hline \multirow[t]{3}{*}{ Lugar de convivencia con 1 a 5 personas fumadoras } & Casa & 42.0 \\
\hline & Aula & 19.35 \\
\hline & Trabajo & 8.4 \\
\hline \multirow[t]{2}{*}{ Molestia por que fumen cerca de ellos } & Sí & 62.2 \\
\hline & No & 37.8 \\
\hline \multirow[t]{2}{*}{ Consumo de tabaco en lugares públicos } & Sí & 2.5 \\
\hline & No & 17.6 \\
\hline \multirow[t]{2}{*}{ Consumo de tabaco durante la enfermedad } & Sí & 3.4 \\
\hline & No & 52.9 \\
\hline
\end{tabular}

FUENTE: Relación entre estrés académico y consumo de alcohol y tabaco en estudiantes de educación superior ( $n=119$ ), septiembre-diciembre 2017.

Relación entre estrés académico y consumo de alcohol y tabaco

No se encontró relación estadísticamente significativa entre las variables estrés académico y consumo de alcohol ( $r s=.141$; $p=.127) ; y$, estrés académico y consumo de tabaco ( $r s=-.069 ; p=.455)$. Por tal motivo, no se puede rechazar la hipótesis nula, entendiéndose que no existe relación entre el estrés académico y el consumo de alcohol y tabaco. Se descubrió que en niveles moderados de estrés académico la mitad de la población presentaba un consumo de riesgo de alcohol (51.3\%). Con respecto al consumo de tabaco, se encontró que en niveles moderados de estrés académico, más de la tercera parte de los alumnos estudiados eran fumadores acti$\operatorname{vos}(34.5 \%)$.

\section{| | | | | | | | | | | | | | | | | | | | | | | | | | | | | | | | | | | | | | | | | | | | | | | | | | | | | | | | | | | | | | | | | | | | | | | | | | | | | | | | | | | | | | | | | | | | |}

\section{Discusión}

En su mayoría, la población seleccionada para el análisis de resultados fueron mu- jeres jóvenes y solteras, cuyo promedio de edad fue 19.8 años, por lo que se encuentra similitud con la investigación rea- 
lizada por Castillo Ávila y cols., en el que el $89.6 \%$ lo conformaban mujeres con una edad promedio de 20.2 años; ambas poblaciones estudiadas cursando educación superior y características sociodemográficas similares. ${ }^{10}$

Los resultados del presente estudio también coinciden con Castillo Ávila y cols., dado que el nivel de estrés académico que más se presentó en la población estudiada fue el moderado. En relación con la dimensión de estresores "la evaluación de profesores" es la principal fuente de estrés por la que pasan los alumnos durante el semestre. ${ }^{10}$

De igual manera, la dimensión de síntomas, se coincide en que la somnolencia y el dolor de cabeza o migraña, son de las principales reacciones físicas presentes en la población de estudio durante los periodos de estrés a los que se ven sometidos, mientras que las reacciones psicológicas que se presentan con mayor frecuencia son los problemas de concentración y ansiedad, angustia o desesperación. Las reacciones comportamentales más habituales en la población fueron el aumento o disminución del consumo de alimentos. Este estudio difiere en cuanto a las estrategias de afrontamiento utilizadas, dado que las principales en nuestra población fueron el "concentrarse en resolver la situación que les preocupa" y "la ventilación de los problemas". La literatura plantea que el adolescente y adulto joven, al ingresar a una institución de educación superior, adquiere varias habilidades, tales como la toma de decisiones, adecuada distribución del tiempo personal, resolución de problemas, entre otras.

En su mayoría, la población de estudio obtuvo un patrón de consumo de alcohol de riesgo, por lo que se concuerda con $\mathrm{Vi-}$ llegas Pantoja y cols, ya que a pesar de que los grupos de estudio presentaban características diferentes, mostraron semejanzas en cuestión al consumo de alcohol. Se considera que, cuando hay consumo de riesgo, es probable que todavía no se presenten trastornos por el consumo de bebidas alcohólicas; sin embargo, existe un aumento del riesgo de consecuencias adversas para el bebedor y los que lo rodean. Se difiere con Villegas Pantoja y cols, en la prevalencia del consumo de tabaco alguna vez en la vida, dado que observaron que más de la mitad lo ha consumido por lo menos alguna vez. ${ }^{3}$

Los resultados demuestran que no existe una relación estadísticamente significativa entre el estrés académico y el consumo de alcohol y tabaco, por lo que no es posible rechazar la hipótesis nula; lo antes mencionado puede atribuirse al momento de la aplicación de los instrumentos ya que no se consideró que la aplicación de éstos fuera en un momento académicamente estresante, que la población estudiantil fuera mixta, y la inclusión de otras variables como el empleo, hijos, lugar de procedencia, entre otras.

En término generales, la totalidad de la población de estudio fue del sexo femenino, la mayoría solteras, las cuales declararon haber tenido momentos de preocupación o nerviosismo en lo transcurrido del semestre, presentando un nivel moderado de estrés.

Estos escenarios estresantes producen en las estudiantes somnolencia o mayor necesidad de dormir, dolor de cabeza y migraña, problemas de concentración, ansiedad y angustia, aumento o reducción del consumo de alimentos y desgano, por lo que emplean como estrategias de afrontamiento la concentración en la resolución de la situación que les preocupa.

Más de la mitad de las estudiantes muestra un consumo de riesgo de alcohol; sin embargo, un porcentaje importante no experimenta consecuencias adversas en el consumo. Respecto al consumo de tabaco, más de un tercio de la población estudiada pertenece al grupo de los fumadores activos, iniciándose en éste a edades tempranas. 
La actual investigación posee algunas limitantes dada la naturaleza transversal del estudio que impide identificar la causalidad entre estas variables, por lo tanto, se invita a continuar investigando la posibilidad de que el estrés académico sea un factor de riesgo para el consumo de alcohol y de tabaco, pero también que el consumo de estas sustancias psicoactivas pueda involucrar situaciones estresantes. Por esta razón, es preciso realizar investigaciones de tipo longitudinal que permitan comprender la interacción de estas variables.

\section{Conclusiones}

El estudio buscó la relación entre las variables de estrés académico, consumo de alcohol y de tabaco, cuyos resultados mostraron que no existe una relación estadísticamente significativa entre estas variables. Sin embargo, se observa una relación de tendencia proporcional, en donde a medida que aumenta el nivel de estrés incrementa el patrón de consumo de alcohol y tabaco, esta situación trae implicaciones graves de salud. Se deben realizar acciones hacia la prevención primaria, principalmente en la población vulnerable, con la finalidad de que se adopten estrategias eficaces de afrontamiento, favoreciendo de esta manera a que la población estudiantil mantenga o mejore su situación de salud.

\section{Bibliografía}

Barraza A. Un modelo conceptual para el estudio del estrés académico. Rev de Psicología de Iztacala. Universidad Nacional Autónoma de México. [Internet]. México; 2006. [Citado el 1 febrero 2018]; 9 (3). Disponible en: http://www.revistas.unam.mx/ index.php/repi/article/download/19028/18052

1. Hernández J, Pozo C. Polo A. La ansiedad ante los exámenes. Un programa para su tratamiento de forma eficaz. [Internet]. Valencia: Promolibro. 2007 [Citado el 1 febrero 2018]. Disponible en: http:// www.apuntesdepsicologia.es/index.php/revista/article/viewFile/117/119

2. Villegas $M$, Alonso M, Alonso B, Guzmán F. Eventos estresantes y la relación con el consumo de alcohol y tabaco en adolescentes. [Internet]. 2014 abr [Citado el 1 febrero 2018]; 20(1): 35-46. Disponible en: https://scielo.conicyt.cl/scielo.php?pid=S07179553 2014000100004\&script =sci_arttext

3. Secretaria de Salud. Encuesta Nacional de Adicciones 2011. [Internet]. México; 2012. [Citado el 1 fbrero 2018]. Disponible en: http://encuestas.insp.mx/ ena/ena2011/ENA2011_alcohol.pdf

4. Babor T. Higgins J. Saunders J. Monteiro M. Cuestionario de Identificación de los Transtornos debidos al Consumo de Alcohol Pautas para su utilización en Atención Primaria. Organización Mundial de la Salud, Departamento de salud mental y dependencia de sustancias. [Internet]. [Citado el 29 de marzo de 2018]. Disponible en: http://www.who.int/substance_abuse/activities/en/AUDITmanualSpanish.pdf
5. Secretaria de Salud. Encuesta Nacional de Consumo de Drogas en Estudiantes 2014: Reporte de Alcohol. [Internet]. Primera edición, México; 2015. [Citado el 12 marzo 2018]. Disponible en: http://www.conadic.salud.gob.mx/pdfs/investigacion/ENCODE_ALCOHOL_2014.pdf

6. Encuesta Global de Tabaquismo en Adultos. Encuesta Global de Tabaquismo en Adultos, GATS México 2015 [Internet]. Insp.mx. 2015 [Citado el 11 febrero 2018]. Disponible en: http://omextad.salud.gob. $\mathrm{mx} /$ contenidos/encuestas/gats2015/ENCUESTA_ GATS 2015.pdf

7. Diccionario Océano Uno Color. Tabaquismo. Barcelona, España: 2010. Pág.40.

8. Secretaria de Salud. Encuesta Nacional de Consumo de Drogas, Alcohol y Tabaco 2016-2017. Reporte de Tabaco. [Internet]. México; 2017. [Citado el 11 febrero 2018]. México, Primera edición 2017. Disponible en: https://drive.google.com/file/d/1lktptv du2nsrSpMBMT4FdqBIk8gikz7q/view

9. Castillo I. Barrios A. Carvajal M. Estrés académico y consumo de alcohol en estudiantes de enfermería de Cartagena. Biblioteca virtual: Universidad de Cartagena. [Internet]. 2015 [Citado el 12 de abril 2018]. Disponible en: http://190.242.62.234:8080/ jspui/bitstream/11227/2653/1/INFORME\% 20 FINAL - \% 20ESTRES \% 20Y\% 20CONSUMO \% 20 DE\% 20ALCOHOL.pdf

10. Barraza A. Propiedades psicométricas del Inventario SISCO del estrés académico. [Internet]. Revista PsicologiaCientifica.com, 9(10). [Citado el 20 de abril 
del 2018] Disponible en: http://www.psicologiacientifica.com/sisco-propiedades-psicometricas

11. Sáiz, P. Portilla M. Paredes M. Bascarán M. Bobes J. Instrumentos de evaluación en alcoholismo. [Internet]. Área de Psiquiatría. Facultad de Medicina. Universidad de Oviedo. 2002. [Internet]. [Citado el 12 de abril 2018]. Disponible en: http://www.fisac.org. $\mathrm{mx} /$ instrumentos $\% 20 \mathrm{de} \% 20$ evaluacion $\% 20$ en $\% 20$ alcoholismo.pdf

12. Comisión Nacional Contra las Adicciones. Escala de consumo de tabaco. 2008 - 2011. [Internet]. México: CONADIC. [Citado el 15 de enero del 2018]. Disponible en: http://www.uv.es/lisis/instrumentos09mexic.htm\#tres

13. Secretaria de Salud. Consejo Nacional Contra las Adicciones. Metodología para la elaboración de estudios epidemiológicos a nivel nacional y local y estudios para grupos especiales relacionados con las adicciones. [Internet]. México: CONADIC. [Citado el 22 de febrero del 2018]. Disponible en: http:// www.conadic.salud.gob.mx/pdfs/publicaciones/ obs02met.pdf

14. Secretaria de Salud. Ley general de salud en materia de investigación para la salud. [Internet]. [Citada el 31 de marzo del 2018] Disponible en: http://www. salud.gob.mx/unidades/cdi/nom/compi/rlgsmis. html

15. Comisión Nacional de Arbitraje Médico. Declaración De Helsinki De La Asociación Médica Mundial. [Internet]. [Citada el 31 de marzo del 2018]. Disponible en: http://www.conamed.gob.mx/prof_salud/pdf/ helsinki.pdf 


\section{ANEXOS}

\section{Anexo I. Instrumento de estrés académico}

\section{Datos sociodemográficos}

Estado civil:

Semestre:

Grupo:

Edad:

\section{INSTRUMENTO DE ESTRÉS ACADÉMICO}

\section{INVENTARIO SISCO DEL ESTRÉS ACADÉMICO}

1.- Durante el transcurso de este semestre, ¿has tenido momentos de preocupación o nerviosismo?

$\square$ Sí

En caso de seleccionar la alternativa "no", el cuestionario se da por concluido, en caso de seleccionar la alternativa "sí, pasar a la pregunta número dos y continuar con el resto de las preguntas.

2.- Con la idea de obtener mayor precisión y utilizando una escala del 1 al 5 señala tu nivel de preocupación o nerviosismo, donde (1) es poco y (5) mucho.

\begin{tabular}{|l|l|l|l|l|}
\hline 1 & 2 & 3 & 4 & 5 \\
\hline & & & & \\
\hline
\end{tabular}

\section{Dimensión estresores}

En el siguiente cuadro señala con una $\mathbf{X}$ con qué frecuencia te inquietaron las siguientes situaciones:

\begin{tabular}{|c|c|c|c|c|c|}
\hline & Nunca & $\begin{array}{l}\text { Rara } \\
\text { vez }\end{array}$ & $\begin{array}{l}\text { Algunas } \\
\text { veces }\end{array}$ & $\begin{array}{c}\text { Casi } \\
\text { siempre }\end{array}$ & Siempre \\
\hline 3.1.- La competencia con los compañeros del grupo & & & & & \\
\hline 3.2.- Sobrecarga de tareas y trabajos escolares & & & & & \\
\hline 3.3.- La personalidad y el carácter del profesor & & & & & \\
\hline $\begin{array}{l}\text { 3.4.- Las evaluaciones de los profesores (exámenes, } \\
\text { ensayos, trabajos de investigación, etc.) }\end{array}$ & & & & & \\
\hline 3.5.- Problemas con el horario de clases & & & & & \\
\hline $\begin{array}{l}\text { 3.6.- El tipo de trabajo que te piden los profesores } \\
\text { (consulta de temas, fichas de trabajo, ensayos, } \\
\text { mapas conceptuales, etc.) }\end{array}$ & & & & & \\
\hline $\begin{array}{l}\text { 3.7.- No entender los temas que se abordan en la } \\
\text { clase. }\end{array}$ & & & & & \\
\hline $\begin{array}{l}\text { 3.8.- Participación en clase (responder a preguntas, } \\
\text { exposiciones, etc.) }\end{array}$ & & & & & \\
\hline 3.9.- Tiempo limitado para hacer el trabajo & & & & & \\
\hline $\begin{array}{l}\text { Otra } \\
\text { (Especifique) }\end{array}$ & & & & & \\
\hline
\end{tabular}




\section{4.- Dimensión síntomas (reacciones)}

En el siguiente cuadro señala con una $\mathbf{X}$ con qué frecuencia tuviste las siguientes reacciones físicas, psicológicas y comportamentales cuando estabas preocupado o nervioso.

\begin{tabular}{|c|c|c|c|c|c|}
\hline \multicolumn{6}{|c|}{ Subdimensión: síntomas o reacciones físicas } \\
\hline & Nunca & $\begin{array}{c}\text { Rara } \\
\text { vez }\end{array}$ & $\begin{array}{l}\text { Algunas } \\
\text { veces }\end{array}$ & $\begin{array}{c}\text { Casi } \\
\text { siempre }\end{array}$ & Siempre \\
\hline \multicolumn{6}{|l|}{$\begin{array}{l}\text { 4.1.- Trastornos en el sueño (insomnio o } \\
\text { pesadillas) }\end{array}$} \\
\hline \multicolumn{6}{|l|}{ 4.2.- Fatiga crónica (cansancio permanente) } \\
\hline \multicolumn{6}{|l|}{ 4.3.- Dolores de cabeza o migrañas } \\
\hline \multicolumn{6}{|l|}{$\begin{array}{l}\text { 4.4.- Problemas de digestión, dolor abdominal o } \\
\text { diarrea }\end{array}$} \\
\hline \multicolumn{6}{|l|}{ 4.5.- Rascarse, morderse las uñas, frotarse, etc. } \\
\hline \multicolumn{6}{|l|}{ 4.6.- Somnolencia o mayor necesidad de dormir } \\
\hline \multicolumn{6}{|c|}{ Subdimensión: síntomas o reacciones psicológicas } \\
\hline & Nunca & $\begin{array}{c}\text { Rara } \\
\text { vez }\end{array}$ & $\begin{array}{l}\text { Algunas } \\
\text { veces }\end{array}$ & $\begin{array}{c}\text { Casi } \\
\text { siempre }\end{array}$ & Siempre \\
\hline \multicolumn{6}{|l|}{$\begin{array}{l}\text { 4.7.- Inquietud (incapacidad de relajarse y estar } \\
\text { tranquilo) }\end{array}$} \\
\hline \multicolumn{6}{|l|}{$\begin{array}{l}\text { 4.8.- Sentimientos de depresión y tristeza } \\
\text { (decaído) }\end{array}$} \\
\hline \multicolumn{6}{|l|}{ 4.9.- Ansiedad, angustia o desesperación. } \\
\hline \multicolumn{6}{|l|}{ 4.10.- Problemas de concentración } \\
\hline \multicolumn{6}{|l|}{$\begin{array}{l}\text { 4.11.- Sentimiento de agresividad o aumento de } \\
\text { irritabilidad }\end{array}$} \\
\hline \multicolumn{6}{|c|}{ Subdimensión: síntomas o reacciones comportamentales } \\
\hline & Nunca & $\begin{array}{l}\text { Rara } \\
\text { vez }\end{array}$ & $\begin{array}{l}\text { Algunas } \\
\text { veces }\end{array}$ & $\begin{array}{c}\text { Casi } \\
\text { siempre }\end{array}$ & Siempre \\
\hline \multicolumn{6}{|l|}{$\begin{array}{l}\text { 4.12.- Conflictos o tendencia a polemizar } \circ \\
\text { discutir }\end{array}$} \\
\hline \multicolumn{6}{|l|}{ 4.13.- Aislamiento de los demás } \\
\hline \multicolumn{6}{|l|}{$\begin{array}{l}\text { 4.14.- Desgano para } \\
\text { escolares }\end{array}$} \\
\hline \multicolumn{6}{|l|}{$\begin{array}{l}\text { 4.15.- Aumento o reducción del consumo de } \\
\text { alimentos }\end{array}$} \\
\hline Otra & & & & & \\
\hline
\end{tabular}




\section{5.- Dimensión estrategias de afrontamiento}

En el siguiente cuadro señala con una $\mathbf{X}$ con qué frecuencia utilizaste las siguientes estrategias para enfrentar la situación que te causaba la preocupación o el nerviosismo.

\begin{tabular}{|c|c|c|c|c|c|}
\hline & Nunca & $\begin{array}{l}\text { Rara } \\
\text { vez }\end{array}$ & $\begin{array}{l}\text { Algunas } \\
\text { veces }\end{array}$ & $\begin{array}{l}\text { Casi } \\
\text { siempre }\end{array}$ & Siempre \\
\hline $\begin{array}{l}\text { 5.1.- Habilidad asertiva (defender nuestras } \\
\text { preferencias, ideas o sentimientos sin dañar a } \\
\text { otros) }\end{array}$ & & & & & \\
\hline $\begin{array}{l}\text { 5.2.- Elaboración de un plan y ejecución de } \\
\text { sus tareas }\end{array}$ & & & & & \\
\hline $\begin{array}{l}\text { 5.3.- Concentrarse en resolver la situación } \\
\text { que me preocupa }\end{array}$ & & & & & \\
\hline 5.4.- Elogios a sí mismo & & & & & \\
\hline $\begin{array}{l}\text { 5.5.- La religiosidad (oraciones o asistencia a } \\
\text { misa) }\end{array}$ & & & & & \\
\hline $\begin{array}{l}\text { 5.6.- Búsqueda de información sobre la } \\
\text { situación }\end{array}$ & & & & & \\
\hline $\begin{array}{l}\text { 5.7.- Fijarse o tratar de obtener lo positivo de } \\
\text { la situación que preocupa }\end{array}$ & & & & & \\
\hline $\begin{array}{l}\text { 5.8.- Ventilación y confidencias (verbalización } \\
\text { de la situación que preocupa) }\end{array}$ & & & & & \\
\hline Otra & & & & & \\
\hline (Especifique) & & & & & \\
\hline
\end{tabular}




\section{Anexo II. Instrumento de alcohol AUDIT (Alcohol Use Disorders Identification Test)}

Instrucciones. Lea cuidadosamente las preguntas. Marque con una $\mathbf{X}$ su respuesta.

1. ¿Con qué frecuencia consume alguna bebida alcohólica?

(0) Nunca (Pase a las preguntas 9-10)

(1) Una o menos veces al mes

(2) De 2 a 4 veces al mes

(3) De 2 a 3 veces a la semana

(4) 4 o más veces a la semana
6. ¿Con qué frecuencia en el curso del último año ha necesitado beber en ayunas para recuperarse después de haber bebido mucho el día anterior?
(0) Nunca
(1) Menos de una vez al mes
(2) Mensualmente
(3) Semanalmente
(4) A diario o casi a diario

2. ¿Cuántas consumiciones de bebidas alcohólicas suele realizar en un día de consumo normal?

(0) 1 ० 2

(1) $3 \circ 4$

(2) $5 \circ 6$

(3) $7,8,09$

(4) 10 o más

3. ¿Con qué frecuencia toma 6 o más bebidas alcohólicas en un solo día?

(0) Nunca

(1) Menos de una vez al mes

(2) Mensualmente

(3) Semanalmente

(4) A diario o casi a diario

Pase a las preguntas 9 y 10 sí la suma total de las preguntas 2 y $3=0$

4. ¿Con qué frecuencia en el curso del último año ha sido incapaz de parar de beber una vez había empezado?
(0) Nunca
(1) Menos de una vez al mes
(2) Mensualmente
(3) Semanalmente
(4) A diario o casi a diario

5. ¿Con qué frecuencia en el curso del último año no pudo hacer lo que se esperaba de usted porque había bebido?

(0) Nunca

(1) Menos de una vez al mes

(2) Mensualmente

(3) Semanalmente

(4) A diario o casi a diario
7. ¿Con qué frecuencia en el curso del último año ha tenido remordimientos o sentimientos de culpa después de haber bebido?
(0) Nunca
(1) Menos de una vez al mes
(2) Mensualmente
(3) Semanalmente
(4) A diario o casi a diario

8. ¿Con qué frecuencia en el curso del último año no ha podido recordar lo que sucedió la noche anterior porque había estado bebiendo?

(0) Nunca

(1) Menos de una vez al mes

(2) Mensualmente

(3) Semanalmente

4) A diario o casi a diario

9. ¿Usted o alguna otra persona ha resultado herido porque usted había bebido?

(0) No

(2) Sí, pero no en el curso del último año

(4) Sí, el último año

10. ¿Algún familiar, amigo, médico o profesional sanitario ha mostrado preocupación por su consumo de bebidas alcohólicas o le han sugerido que deje de beber?

(0) No

(2) Sí, pero no en el curso del último año

(4) Sí, el último año 
Anexo III. Instrumento de tabaco

\section{EECT (Encuesta Exploratoria del Consumo de Tabaco)}

Instrucciones: Contesta lo que se te pide y marca con una " $X$ " sólo una de las opciones que se te presentan.

\begin{tabular}{|c|c|c|}
\hline 1. ¿Alguna vez has fumado? & $\begin{array}{l}\text { a) } \\
\text { b) }\end{array}$ & $\begin{array}{l}\text { Sí } \\
\text { No }\end{array}$ \\
\hline $\begin{array}{l}\text { 2. En los últimos } 12 \text { meses, ¿has } \\
\text { fumado tabaco? }\end{array}$ & $\begin{array}{l}\text { a) } \\
\text { b) } \\
\text { c) }\end{array}$ & $\begin{array}{l}\text { Sí } \\
\text { No } \\
\text { Nunca he fumado tabaco }\end{array}$ \\
\hline $\begin{array}{l}\text { 3. En los últimos } 30 \text { días, ¿has } \\
\text { fumado tabaco? }\end{array}$ & $\begin{array}{l}\text { a) } \\
\text { b) } \\
\text { c) } \\
\text { d) } \\
\text { e) }\end{array}$ & $\begin{array}{l}\text { No } \\
\text { Sí, de 1-5 días } \\
\text { Sí, de 6-19 días } \\
\text { Sí } 20 \text { días o más } \\
\text { Nunca he fumado tabaco }\end{array}$ \\
\hline $\begin{array}{l}\text { 4. En toda tu vida, ¿has fumado más } \\
\text { de } 100 \text { cigarros, es decir, más de } \\
5 \text { cajetillas? }\end{array}$ & $\begin{array}{l}\text { a) } \\
\text { b) } \\
\text { c) }\end{array}$ & $\begin{array}{l}\text { Sí } \\
\text { No } \\
\text { Nunca he fumado tabaco }\end{array}$ \\
\hline $\begin{array}{l}\text { 5. ¿Cuántos años tenías cuando } \\
\text { fumaste por primera vez tabaco? }\end{array}$ & $\begin{array}{l}\text { a) } \\
\text { b) } \\
\text { c) } \\
\text { d) } \\
\text { e) } \\
\text { f) } \\
\text { g) } \\
\text { h) } \\
\text { i) } \\
\text { j) } \\
\text { n) }\end{array}$ & $\begin{array}{l}\text { Menos de } 6 \\
\text { De } 6 \text { a } 8 \\
\text { De } 9 \text { a } 10 \\
11 \text { años } \\
12 \text { años } \\
13 \text { años } \\
14 \text { años } \\
15 \text { años } \\
16 \text { años } \\
17 \text { años o más } \\
\text { Nunca he fumado tabaco }\end{array}$ \\
\hline $\begin{array}{l}\text { 6. ¿Con qué frecuencias fumas o } \\
\text { fumabas cigarrillos? }\end{array}$ & $\begin{array}{l}\text { a) } \\
\text { b) } \\
\text { c) } \\
\text { d) } \\
\text { e) } \\
\text { f) }\end{array}$ & $\begin{array}{l}\text { Diariamente } \\
\text { Semanalmente } \\
\text { Mensualmente } \\
\text { Ocasionalmente } \\
\text { Al menos una vez al año } \\
\text { Nunca he fumado tabaco }\end{array}$ \\
\hline $\begin{array}{l}\text { 7. ¿Alguna vez has intentado dejar } \\
\text { de fumar? }\end{array}$ & $\begin{array}{l}\text { a) } \\
\text { b) } \\
\text { c) }\end{array}$ & $\begin{array}{l}\text { Sí } \\
\text { No } \\
\text { Nunca he fumado tabaco }\end{array}$ \\
\hline $\begin{array}{l}\text { 8. De las personas con las que } \\
\text { convives diario, ¿cuántas fuman? }\end{array}$ & $\begin{array}{l}\text { a) } \\
\text { b) } \\
\text { c) } \\
\text { d) }\end{array}$ & $\begin{array}{l}\text { En el interior de su casa } \\
\text { En el salón de clases } \\
\text { En el lugar de trabajo } \\
\text { Ninguna }\end{array}$ \\
\hline $\begin{array}{l}\text { 9. ¿Te molesta que fumen cerca de } \\
\text { ti? }\end{array}$ & $\begin{array}{l}\text { a) } \\
\text { b) }\end{array}$ & $\begin{array}{l}\text { Sí } \\
\text { No }\end{array}$ \\
\hline $\begin{array}{l}\text { 10. ¿Cuántos cigarros fumas al } \\
\text { día? }\end{array}$ & $\begin{array}{l}\text { a) } \\
\text { b) } \\
\text { c) } \\
\text { d) }\end{array}$ & $\begin{array}{l}\text { De } 1 \text { a } 15 \text { cigarrillos } \\
\text { De } 16 \text { a } 25 \text { cigarrillos } \\
26 \text { o más cigarrillos diarios } \\
\text { No fumo diario }\end{array}$ \\
\hline
\end{tabular}




\begin{tabular}{|c|c|c|}
\hline & e) & Nunca he fumado tabaco \\
\hline $\begin{array}{l}\text { 11. ¿En qué momento del día } \\
\text { fumas más? }\end{array}$ & $\begin{array}{l}\text { a) } \\
\text { b) } \\
\text { c) } \\
\text { d) }\end{array}$ & $\begin{array}{l}\text { En las mañanas } \\
\text { En las tardes o al anochecer } \\
\text { No fumo diario } \\
\text { Nunca he fumado tabaco }\end{array}$ \\
\hline $\begin{array}{l}\text { 12. ¿Cuánto tiempo tardas en } \\
\text { encender el primer cigarrillo } \\
\text { después de que te despiertas? }\end{array}$ & $\begin{array}{l}\text { a) } \\
\text { b) } \\
\text { c) } \\
\text { d) }\end{array}$ & $\begin{array}{l}30 \text { minutos o menos } \\
\text { Más de } 30 \text { minutos } \\
\text { No fumo diario } \\
\text { Nunca he fumado }\end{array}$ \\
\hline $\begin{array}{l}\text { 13. ¿Cuál es el cigarrillo que } \\
\text { menos te gustaría dejar pasar } \\
\text { durante el día? }\end{array}$ & $\begin{array}{l}\text { a) } \\
\text { b) } \\
\text { c) } \\
\text { d) }\end{array}$ & $\begin{array}{l}\text { El de la mañana } \\
\text { Otro } \\
\text { No fumo diario } \\
\text { Nunca he fumado }\end{array}$ \\
\hline $\begin{array}{l}\text { 14. ¿Es difícil abstenerse de } \\
\text { fumar en lugares públicos donde } \\
\text { se prohibe? (iglesias, bibliotecas, } \\
\text { cines, secciones de no fumar, } \\
\text { etc.) }\end{array}$ & $\begin{array}{l}\text { a) } \\
\text { b) } \\
\text { c) } \\
\text { d) }\end{array}$ & $\begin{array}{l}\text { Sí } \\
\text { No } \\
\text { No fumo diario } \\
\text { Nunca he fumado tabaco }\end{array}$ \\
\hline $\begin{array}{l}\text { 15. Cuando te enfermas y debes } \\
\text { de permanecer en cama, ¿fumas? }\end{array}$ & $\begin{array}{l}\text { a) } \\
\text { b) } \\
\text { c) }\end{array}$ & $\begin{array}{l}\text { Sí } \\
\text { No } \\
\text { Nunca he fumado tabaco }\end{array}$ \\
\hline
\end{tabular}


Anexo IV. Consentimiento informado

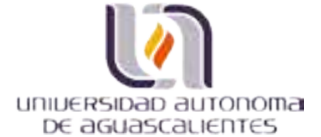

DEIUERSIDAD aUIONOMa
DE aGuascautientes
CENTRO DE CIENCIAS DE LA SALUD

DEPARTAMENTO DE ENFERMERÍA

LICENCIATURA EN ENFERMERÍA

CONSENTIMIENTO INFORMADO

\section{"RELACIÓN ENTRE ESTRÉS ACADÉMICO Y CONSUMO DE ALCOHOL Y TABACO EN ESTUDIANTES DE EDUCACIÓN SUPERIOR"}

Estimado (a) estudiante:

Se le invita a participar en este estudio de investigación referente a la relación entre el estrés académico y el consumo de alcohol y tabaco. Al participar en esta investigación usted no tendrá un beneficio inmediato; sin embargo, al obtener los resultados se conocerá si existe relación entre el estrés académico y el consumo de alcohol y tabaco, si existiera dicha relación se trabajará en crear estrategias en materia de educación y prevención. A partir de esta investigación se busca contribuir en la institución académica para la compresión del fenómeno del consumo o dependencia de alcohol y tabaco, derivado de la exposición a altos niveles de estrés, pues esto puede traducirse en: disminución del rendimiento académico, depresión, deserción universitaria, accidentes de tránsito, lesiones personales y deterioro físico y psicológico. De igual manera, permitirá visualizar la magnitud real del problema, siendo la base de futuras intervenciones por parte de instituciones educativas y de salud para un adecuado manejo de esta situación.

La información que usted proporcione será completamente confidencial, solamente tendrá acceso a ella el equipo de investigación y no se utilizará para ningún otro fin. Usted no estará expuesto a ningún riesgo al participar en la investigación, ni tampoco recibirá ningún pago y no implicará costo alguno por la participación en la misma.

Su participación es voluntaria y está en plena libertad de negarse a participar o a retirarse cuando usted decida. Si usted tiene alguna duda o pregunta mientras contesta la encuesta, los investigadores estarán a su disposición para resolverlas.

Firma fecha 